\title{
VERY HIGH GRADIENT QUADRUPOLES
}

\author{
J. Strait, Fermilab ${ }^{1}$, Batavia, IL 60510, USA
}

\section{Abstract}

High gradient superconducting quadrupoles are an essential component of high energy hadron colliders, both in the arcs and in the insertions. The highest performance quadrupoles today are those for the Large Hadron Collider (LHC) at CERN. These 56 to $70 \mathrm{~mm}$ aperture magnets, developed in Europe, the United States, and Japan, reach gradients on the order of $250 \mathrm{~T} / \mathrm{m}$, which is near the limit for NbTi technology. Designs based on $\mathrm{Nb}_{3} \mathrm{Sn}$ are now being developed, which are aimed at use in second generation LHC low- $\beta$ insertions and in the next generation of very large hadron colliders (VLHC). These quadrupoles are planned to achieve gradients up to $300 \mathrm{~T} / \mathrm{m}$ with the large aperture required for the LHC insertions or $450 \mathrm{~T} / \mathrm{m}$ for smaller aperture arc quadrupoles for VLHC. Even higher gradients may be required for the VLHC final focus.

\section{INTRODUCTION}

This paper presents a survey of the high-gradient superconducting quadrupoles that are utilized in high energy colliders, and that are contemplated for the next generation of colliders. The quadrupoles for the LHC are mature designs based on decades of experience with $\cos 2 \theta$ coils using NbTi superconductor. With pole-tip fields on the order of $8 \mathrm{~T}$ under operating conditions, these magnets are at the practical limit of this technology. The quadrupoles planned for LHC upgrades or the next generation of colliders are relatively straightforward extensions of the current designs, which replace the $\mathrm{NbTi}$ superconductor with $\mathrm{Nb}_{3} \mathrm{Sn}$, but retain the familiar $\cos 2 \theta$ coil geometry and conventional mechanical designs. For specialized applications in the highest energy hadron colliders currently under consideration, designs utilizing high-temperature superconductors (HTS) or nonconventional conductor geometries are also contemplated.

Section 2 summarizes the current state-of-the-art. Some examples of $\mathrm{Nb}_{3} \mathrm{Sn}$ magnets planned as the next generation of quadrupoles, and which will be developed in the coming years, are presented in Section 3. Two unconventional designs, which illustrate the sort of longrange R\&D that will be necessary for very high performance colliders of the future, are shown in Section 4. This is a selective survey intended to summarize the state of high gradient quadrupole development, and is

\footnotetext{
${ }^{1}$ Supported by the U.S. Department of Energy

E-mail: strait@fnal.gov.
}

certainly not comprehensive of all high-performance or innovative designs.

\section{THE CURRENT STATE-OF-THE-ART}

The current state of the art is defined by the insertion and main arc quadrupoles for the LHC. Figure 1 shows cross-sections of three $70 \mathrm{~mm}$ aperture quadrupoles developed for the LHC insertion regions (IR). Two have four-layers coils made of relatively narrow cable, and have a close iron yoke which provides the main structural support. The third has a two-layer coil made from wide cable, and has structural collars and a non-structural yoke.

a)
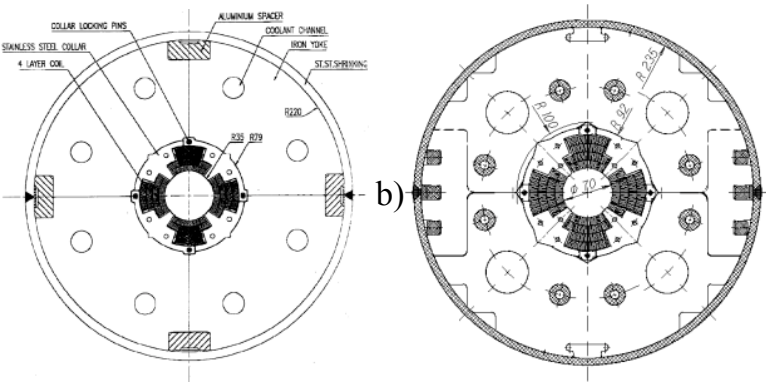

c)

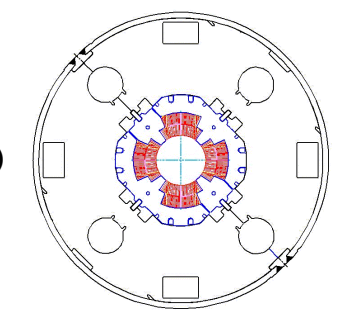

Figure 1: Three quadrupoles developed for the LHC insertions: a) MQY, b) MQXA and c) MQXB.

The first design, called MQY (Fig 1a), was developed by CERN and Oxford Instruments[1], and will be used in a twin-aperture configuration in the LHC insertions. The coil is strongly graded between two cable types, and this is perhaps the most efficient quadrupole design in existence. The quench histories for three single aperture $1 \mathrm{~m}$ models are shown in Fig. 2. This magnet currently holds the world record gradient of $260 \mathrm{~T} / \mathrm{m}$.

The MQXA (Fig 1b) and MQXB (Fig 1c) will make up the final focus inner triplet in the LHC experimental insertions. The MQXA, developed by KEK[2], is similar in design to the MQY, but the coil is less aggressively graded. The first full-scale prototype is shown in Fig 3. Training histories of three $1 \mathrm{~m}$ models quadrupole and of 
the full- scale prototype are shown in Figs. 4 and 5 respectively. The highest gradient reached by this series is $252 \mathrm{~T} / \mathrm{m}$.

The MQXB has been developed by Fermilab[3], who is also responsible for assembling both the KEK quadrupoles and their own into the inner triplet cryostats, as shown in Fig. 6. The quench training for five $1.8 \mathrm{~m}$ models and the full-scale prototype are shown in Fig. 7; the maximum gradient reached was $249 \mathrm{~T} / \mathrm{m}$.

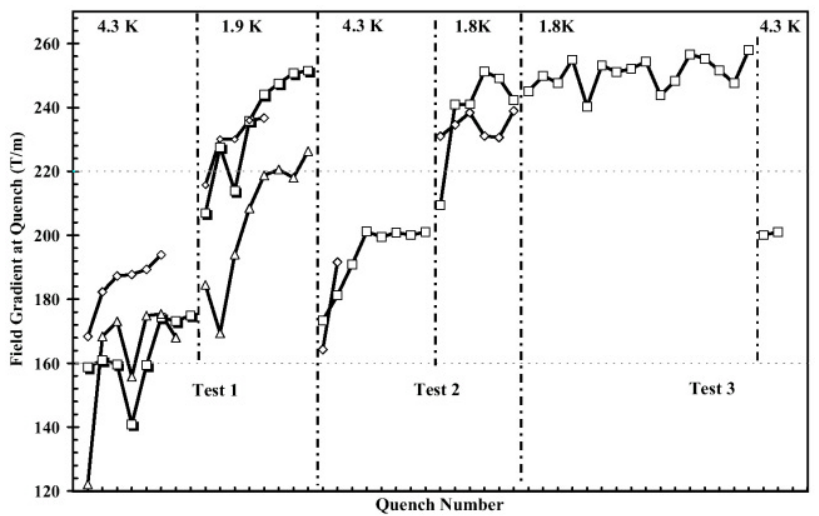

Figure 2: Quench training history of three single aperture MQY model quadrupoles.

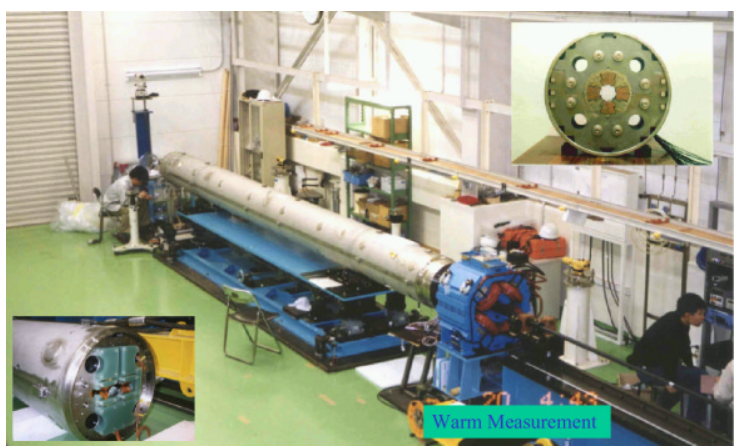

Figure 3: Full-scale prototype MQXB quadrupole at KEK.

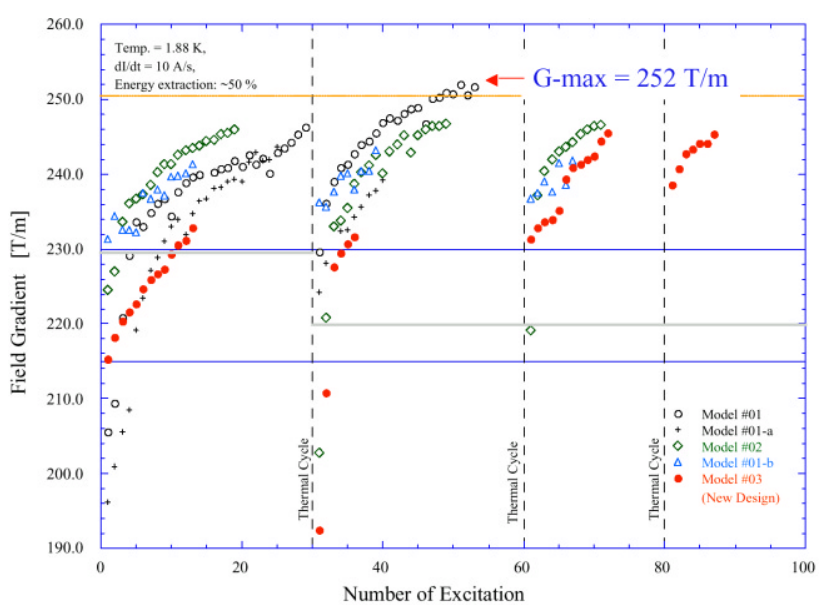

Figure 4: Quench training history of three MQXB model quadrupoles

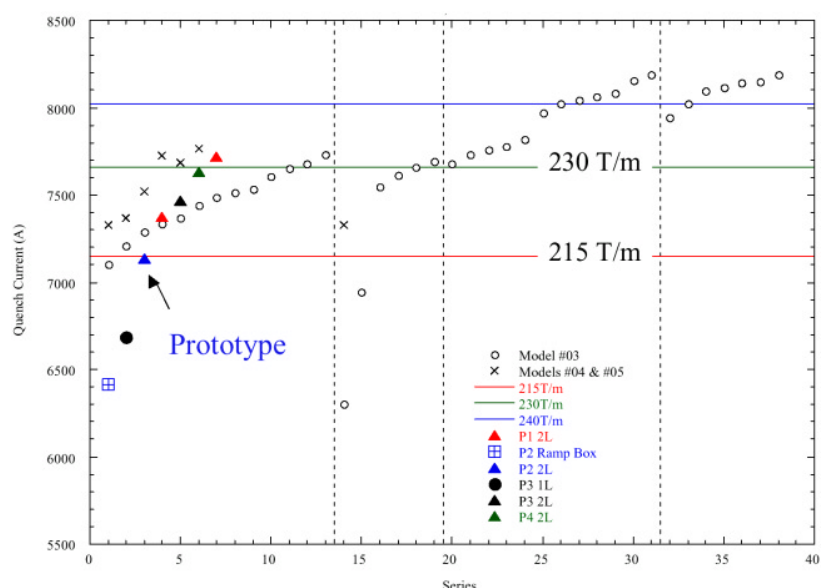

Figure 5: Quench training history of the full scale prototype MQXB quadrupole compared with the model magnets.

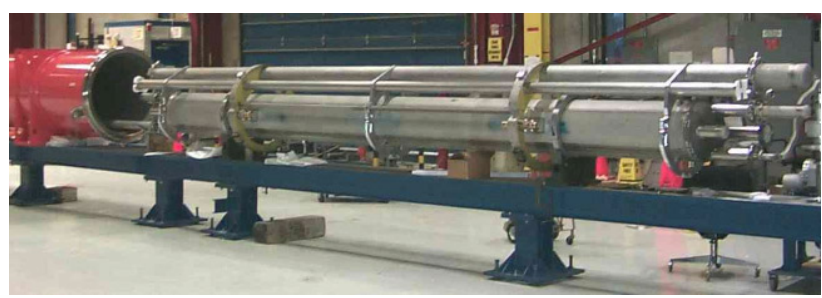

Figure 6: Full-scale prototype MQXB quadrupole being prepared for cryostat insertion at Fermilab.

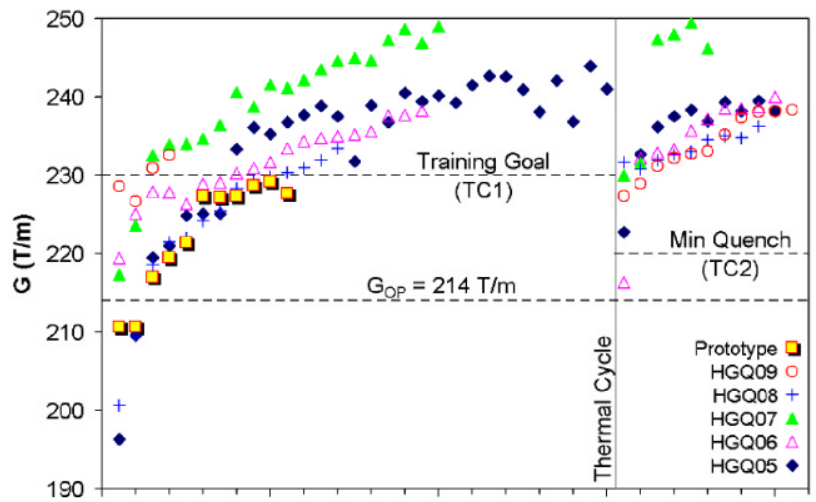

Quench Sequence

Figure 7: Quench training history of five model and one full-scale prototype MQXB quadrupole.

The LHC arc quadrupole, Fig. 8, is a $56 \mathrm{~mm}$ aperture two-in-one magnet, optimized for large-scale production, which has been developed by Saclay and CERN[4]. It is mated to correction coils, beam instrumentation and a cryogenic service module to form a short straight section (SSS). See Fig. 9. The three pre-production quadrupoles easily reach the nominal gradient of $223 \mathrm{~T} / \mathrm{m}$, and, as shown in Fig. 10, reach $240-250 \mathrm{~T} / \mathrm{m}$ after only a few training quenches. 


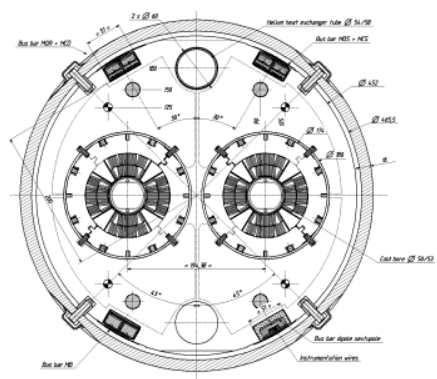

Figure 8: LHC arc quadrupole magnet (MQ).

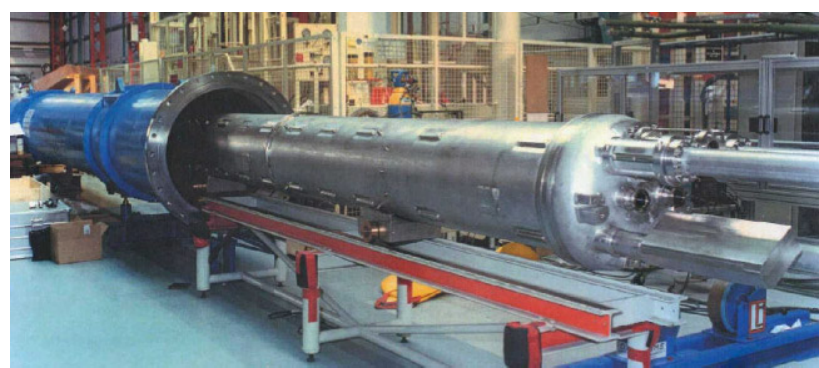

Figure 9: Pre-production LHC short straight section.

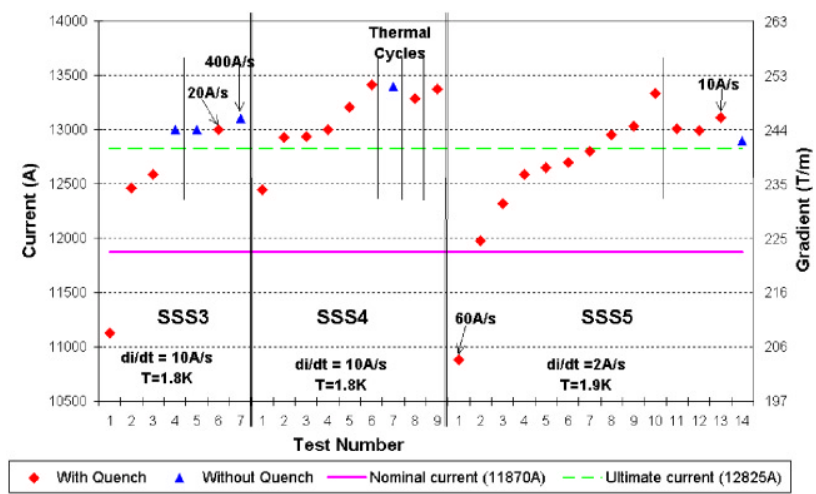

Figure 10: Quench training history of three pre-production LHC arc quadrupoles.

\section{THE NEXT GENERATION}

The quadrupoles described above have essentially reached the limit of what is possible with NbTi conductor. The peak field in the conductor under operating conditions is $8 \mathrm{~T}$ or more, and the short sample limits approach $10 \mathrm{~T}$. They require superfluid helium cooling to achieve the high gradients demanded by LHC. Higher gradients will require the use of higher performance conductor, such as $\mathrm{Nb}_{3} \mathrm{Sn}$. Figure 11[5] shows the substantial progress in the development of this material over the past 17 years. Current densities at $12 \mathrm{~T}$ can now be achieved that are comparable to those reached at half that field in NbTi.

An example of a $\mathrm{Nb}_{3} \mathrm{Sn}$ based quadrupole, which is proposed for the final focus system for TESLA, is shown in Fig. 12[6]. It uses the same coil package design as for the LHC, but replaces the $\mathrm{NbTi}$ cable with $\mathrm{Nb}_{3} \mathrm{Sn}$. Even without an iron yoke, and when imbedded in the $4 \mathrm{~T}$ field of the experimental solenoid, it is designed to

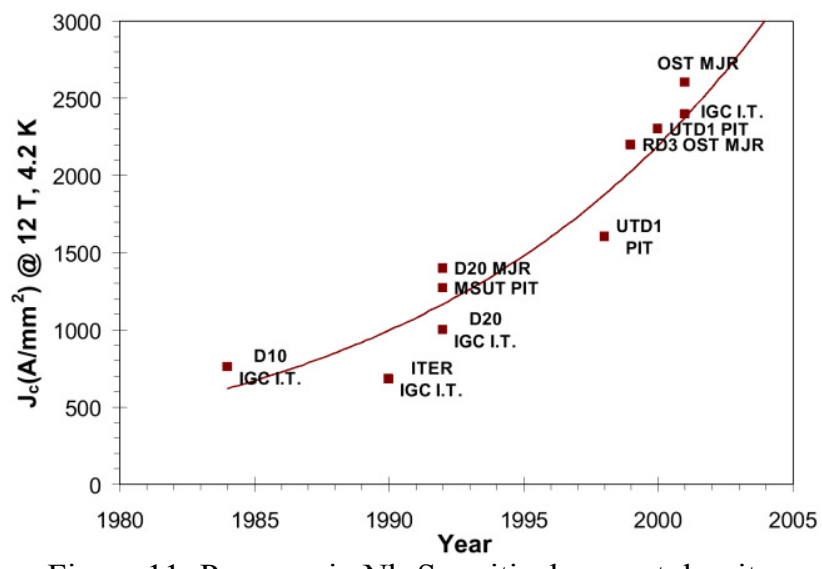

Figure 11: Progress in $\mathrm{Nb}_{3} \mathrm{Sn}$ critical current density.

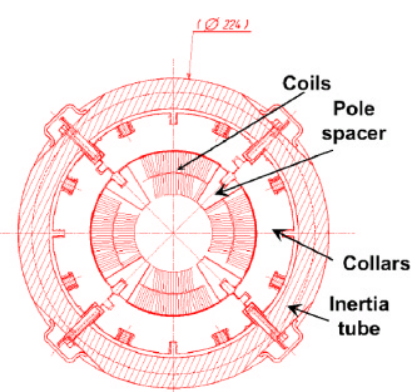

Figure 12: Candidate design for TESLA final focus quadrupole.

operate at $250 \mathrm{~T} / \mathrm{m}$. It requires $\mathrm{J}_{\mathrm{c}}>1800 \mathrm{~A} / \mathrm{mm}^{2}$, well within the range available today.

Another proposed application for $\mathrm{Nb}_{3} \mathrm{Sn}$ quadrupoles is in second generation LHC interaction regions. It is expected that the inner triplets currently under construction at Fermilab and KEK will eventually limit the luminosity of the LHC and will need to be replaced as part of the natural evolution of the machine. The simplest upgrade would be to rebuild the existing inner triplets either with higher gradient or larger aperture quadrupoles. A study presented at this conference concludes[7] that the latter is preferred, as it allows a more substantial decrease in $\beta^{*}$. Figure 13 shows the two candidate quadrupole designs considered. These are two-layer coils similar to that of the MQXB, but with a smaller coil to yoke spacing. Figure 14 shows how the short sample gradient depends on conductor performance. For example, if

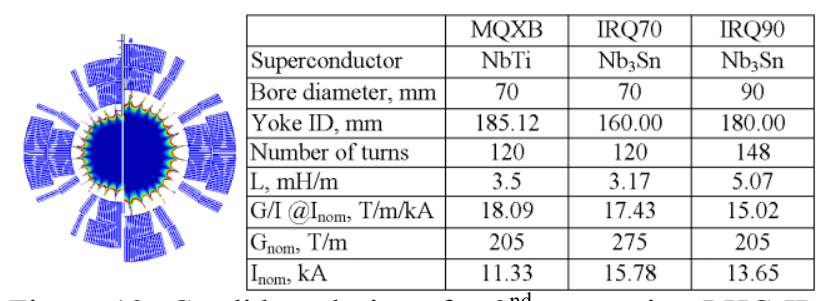

Figure 13: Candidate designs for $2^{\text {nd }}$ generation LHC IR quadrupoles using $\mathrm{Nb}_{3} \mathrm{Sn}$ cable, compared with the MQXB design. 


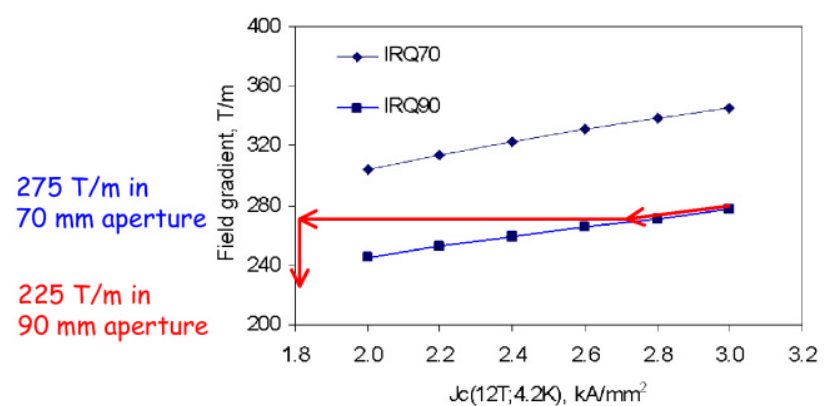

Figure 14: Operating gradients for proposed $70 \mathrm{~mm}$ and $90 \mathrm{~mm}$ aperture $2^{\text {nd }}$ generation LHC IR quadrupoles.

$3000 \mathrm{~A} / \mathrm{mm}^{2}$ is achieved in the wire, then allowing for $10 \%$ cabling degradation (diagonal red arrow), and $20 \%$ operating margin (vertical arrow), an operating gradient of $225 \mathrm{~T} / \mathrm{m}$ can be achieved in a $90 \mathrm{~mm}$ aperture.

Arc quadrupoles for a very large hadron collider (VLHC) with energy above $100 \mathrm{TeV}$ [5] will also require the use of $\mathrm{Nb}_{3} \mathrm{Sn}$. Examples of two designs for a $\sqrt{\mathrm{s}}_{\mathrm{s}}=$ $200 \mathrm{TeV}$ VLHC are shown in Fig. 15[8]. Both operate at $400 \mathrm{~T} / \mathrm{m}$ with a $43.5 \mathrm{~mm}$ aperture. One design, with horizontal beam separation, is focusing for one beam and defocusing for the other. As with the LHC arc quadrupoles, the two apertures are magnetically coupled. The other, with vertical separation, is designed to be focusing or defocusing for both beams, as would be natural to use with flat-beam optics (see below). In this case the two apertures are magnetically decoupled, requiring a larger yoke.

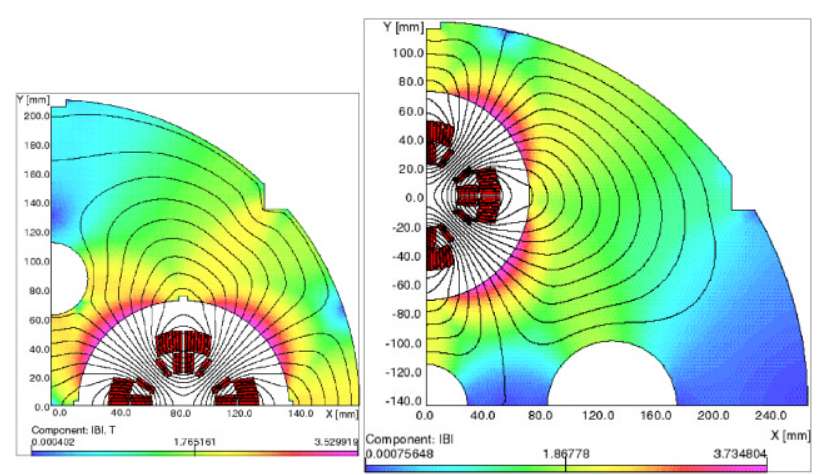

Figure 15: Two candidate designs for a $\sqrt{s}_{\mathrm{s}}=200 \mathrm{TeV}$ VLHC arc quadrupoles for horizontal (left) or vertical (right) beam separation.

\section{BEYOND THE STATE-OF-THE-ART}

The quadrupoles described in section 2 are relatively straightforward extrapolations from familiar designs used in many accelerators, with the use of $\mathrm{Nb}_{3} \mathrm{Sn}$ being the main innovation. The gradients specified can be achieved with conductor that is currently available, or will be in the foreseeable future. The main challenge is to master the difficult technology of working with the brittle A15 material. More speculative designs have been proposed to address specific requirements of hadron collider interaction regions.
An alternate upgrade path to the simple replacement of the existing LHC inner triplets discussed above would be to add a doublet of very high gradient quadrupoles ("Q0") between the existing triplets and the interaction point (IP). An example of the optics for such a case is shown in Fig. 16[9]. Here $\beta^{*}=18 \mathrm{~cm}$ is reached, with $\beta_{\max }$ comparable to that in the existing LHC optics. The inner-most of the pair of Q0 magnets operates at $540 \mathrm{~T} / \mathrm{m}$ with a $50 \mathrm{~mm}$ aperture, which yields a peak field in the conductor of over $16 \mathrm{~T}$. In addition, this magnet would absorb more than $1 \mathrm{~kW}$ of power from the collision debris, since it would displace absorbers that exists in the current IR layout. A design concept using HTS material is sketched in Fig. 17. It is made with conductor-friendly racetrack coils in which the outer blocks are the return path of the conductors near the bore. This coil design makes use of HTS material conceivable. In addition, the active flux return results in a smaller yoke than with a conventional $\cos 2 \theta$ coil design. However, at $500 \mathrm{~mm}$ OD and several meters length, this is not a small magnet and it would be very difficult to integrate this within the forward detectors of the LHC experiments.

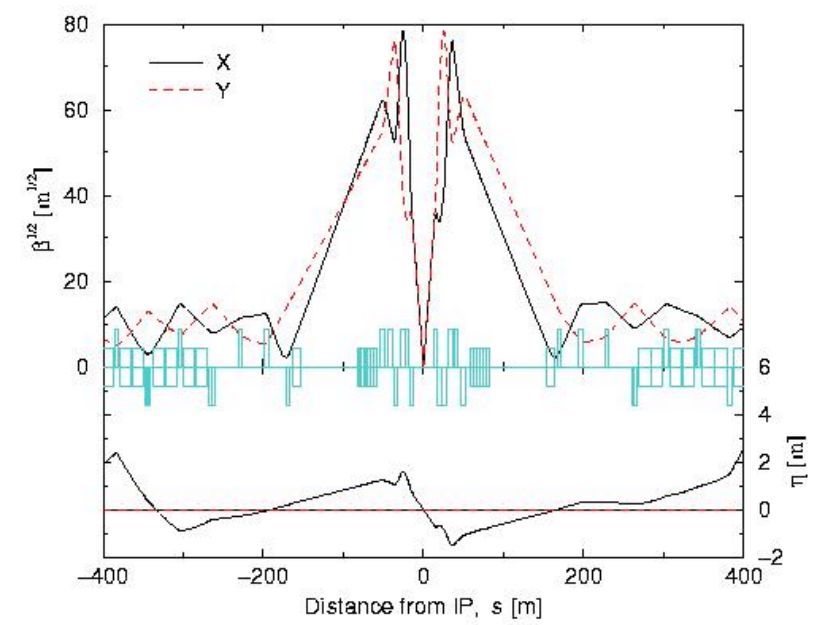

Figure 16: LHC IR optics with "Q0" doublet inserted in between the IP and the existing triplet.

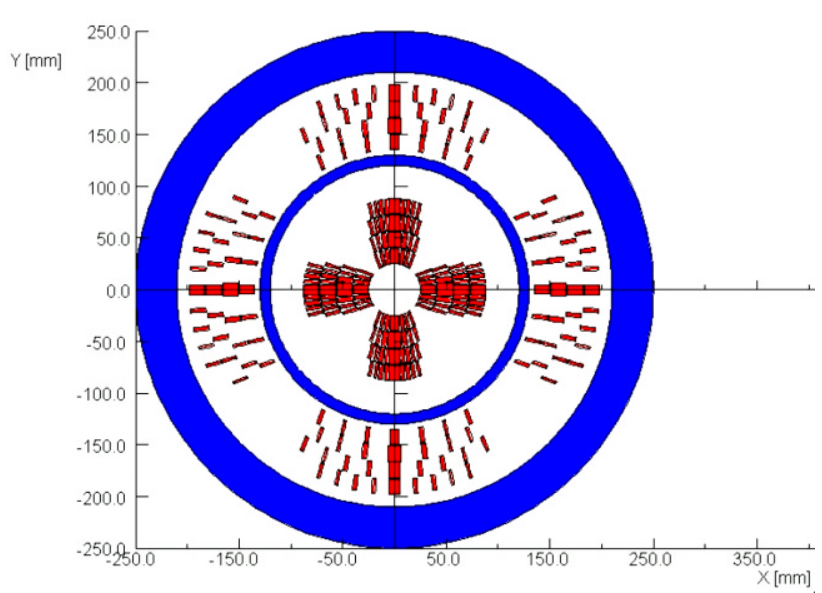

Figure 17: Design concept for an LHC Q0 quadrupole using high-temperature superconductor. 
A VLHC with beam energy above about $50 \mathrm{TeV}$ would be the first synchrotron radiation damped hadron machine, which opens the way to consider flat beams, as is routine in electron machines. To exploit the flat beams at the IP, the quadrupoles must be vertically focusing for both beams on both sides of the IP. This, in turn, requires the use of twin-aperture final focus quadrupoles. To minimize $\beta_{\max }$, the quadrupoles must be as close as possible to the IP and therefore have as small as possible an aperture spacing. Optics for such a case is shown in Fig. 18[5]. The final focus quadrupole doublet is placed between the two beam separation dipoles, and the spacing between apertures is only about $70 \mathrm{~mm}$. Here $\mathrm{Nb}_{3} \mathrm{Sn}$ or HTS material will be required not only due to the high field, but also due to the very high heating from the collision debris (10's of $\mathrm{kW}$ ). A design concept for such a quadrupole, based on $\mathrm{Nb}_{3} \mathrm{Sn}$ racetrack coils and operating at $400 \mathrm{~T} / \mathrm{m}$, is shown in Fig 19[5]. This "design" is only an idea at this stage, and it is clear that it will be very difficult to achieve good field quality and the high gradient required, while containing the enormous forces on the conductor and providing adequate cooling. This illustrates the great challenges that remain if accelerator based particle physics is to advance into the multi-TeV mass range.

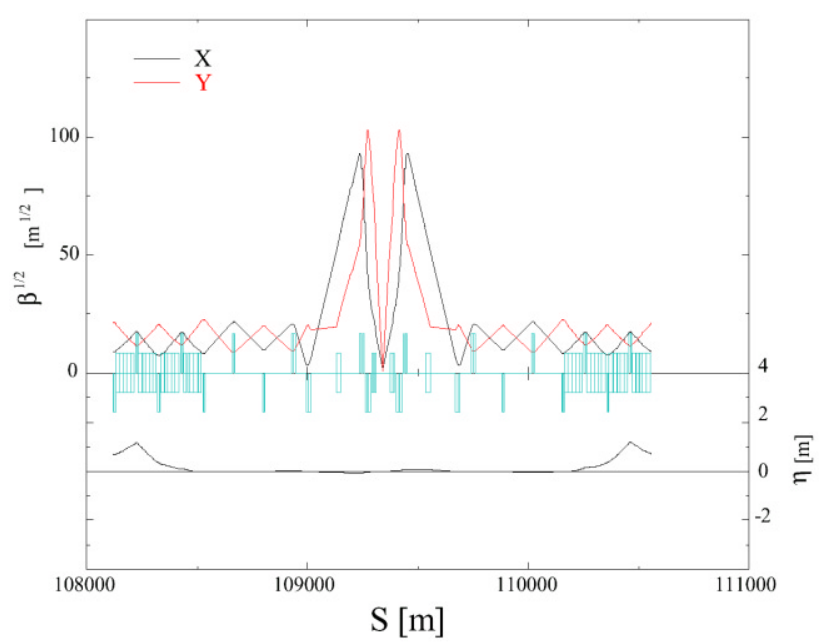

Figure 18: Flat-beam IR optics for a $\sqrt{s}_{\mathbf{s}}=200 \mathrm{TeV}$ VLHC.
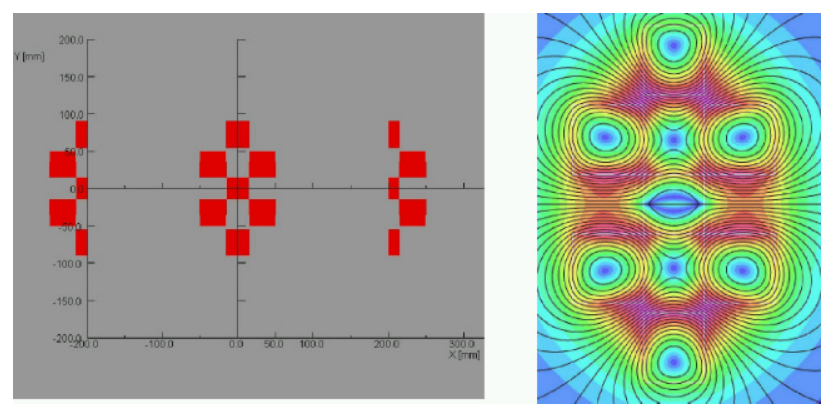

Figure 19: Possible twin-aperture "F-F" IR quadrupole for a VLHC with flat-beam optics.

\section{ACKNOWLEDGEMENTS}

Preparation of this paper benefited greatly from material provide by and discussions with many people, including A. Devred, R. Gupta, J. Kerby, G. Kirby, M. Lamm, R. Ostojic，T. Tortschanoff，A. Yamamoto, and A. Zlobin.

\section{REFERENCES}

[1] M. Lamm et al., "Test of the $70 \mathrm{~mm}$ Quadrupole for the LHC Low-Beta Insertions," EPAC'98, Stockholm.

[2] A. Yamamoto, et al., "Development of LHC Low- $\beta$ Quadrupole Magnets at KEK," PAC 2001, Chicago.

[3] N. Andreev, et al., "Status of the LHC Inner Triplet Quadrupole Program at Fermilab," ASC 2000, Virginia Beach.

[4] T. Tortschanoff, et al., "Performance of Prototypes and Start up of Series Fabrication of the LHC Arc Quadrupoles," PAC 2001, Chicago.

[5] Design Study for a Staged VLHC, Fermilab TM2149, 2001, P. Limon, ed., http://vlhc.org/.

[6] A. Devred, et al., "Conceptual Design for the Final Focus Quadrupole Magnets for TESLA," TESLA2001-17.

[7] T. Sen, et al., "Second Generation High Gradient Quadrupoles for the LHC Interaction Regions," PAC 2001, Chicago.

[8] V.V. Kashikhin and A.V. Zlobin, " $\mathrm{Nb}_{3} \mathrm{Sn}$ Arc Quadrupole Magnets for VLHC," PAC 2001, Chicago.

[9] M Harrison, S. Peggs, and R. Gupta, private communication. 